\title{
Scattering effects in passive foil focusing of ion beams
}

\author{
Albert Yuen* \\ Lawrence Berkeley National Laboratory, Berkeley, California 94720, USA \\ and Department of Nuclear Engineering, UC Berkeley, Berkeley, California 94720, USA \\ Steven M. Lund \\ Facility for Rare Isotope Beams, Michigan State University, East Lansing, Michigan 48824, USA \\ John J. Barnard \\ Lawrence Livermore National Laboratory, Livermore, California 94550, USA \\ Ronald H. Cohen \\ CompX, Del Mar, California 92014, USA \\ Jonathan S. Wurtele \\ Lawrence Berkeley National Laboratory, Berkeley, California 94720, USA \\ and Department of Physics, UC Berkeley, Berkeley, California 94720, USA
}

(Received 30 April 2015; published 11 September 2015)

\begin{abstract}
A stack of thin, closely spaced conducting foils has been investigated by Lund et al. [Phys. Rev. ST Accel. Beams 16, 044202 (2013)] as a passive focusing lens for intense ion beams. The foils mitigate spacecharge defocusing forces to enable the beam self-magnetic field to focus. In this study, we analyze possible degradation of focusing due to scattering of beam ions resulting from finite foil thickness using an envelope model and numerical simulations with the particle-in-cell code WARP. Ranges of kinetic energy where scattering effects are sufficient to destroy passive focusing are quantified. The scheme may be utilized to focus protons produced in intense laser-solid accelerator schemes. As an example, the spot size of an initially collimated $30 \mathrm{MeV}$ proton beam with initial rms radius $200 \mu \mathrm{m}$, perveance $Q=1.8 \times 10^{-2}$, and initial transverse emittance $\varepsilon_{x, \text { rms }}=0.87 \mathrm{~mm}$ mrad propagating through a stack of $6.4 \mu \mathrm{m}$ thick foils, spaced $100 \mu \mathrm{m}$ apart, gives a $127.5 \mu \mathrm{m}$ spot with scattering and a $81.0 \mu \mathrm{m}$ spot without scattering, illustrating the importance of including scattering effects.
\end{abstract}

DOI: 10.1103/PhysRevSTAB.18.091301

PACS numbers: 29.27.Bd, 41.75.-i, 52.59.Sa

\section{INTRODUCTION}

Charged particle beams tend to radially expand under space-charge forces and thermal forces if no applied focusing fields constrain their transverse dynamics [1,2]. Active focusing systems such as solenoids or quadrupole magnets are usually used. However, passive focusing schemes have been investigated which employ material structures to reduce self-fields. These include: Metallic conic guide tubes for electron focusing [3] and ion focusing [4], and stacks of thin foils for electron focusing [5]. Recently, it has been shown that ion beams propagating through a stack of thin metallic foils can be magnetically self-focused due to the mitigation of their electrostatic

\footnotetext{
*ayuen@1bl.gov

Published by the American Physical Society under the terms of the Creative Commons Attribution 3.0 License. Further distribution of this work must maintain attribution to the author(s) and the published article's title, journal citation, and DOI.
}

repulsion [6]. This novel passive focusing scheme opens the possibility of collimating or focusing ion beams to a small spot size since the focusing becomes stronger as the beam radius reduces. Applications may include focusing intense beams on the $\mathrm{X}$-target for fast ignition-driven fusion energy [7], injectors for compact proton accelerators for tumor therapy [8], and ion beam-driven warm dense matter studies [9]. The stack of thin foils can be made from aluminum and manufactured at low cost. Foil stacks can be used for transverse focusing of laser-produced proton beams [10] where intense space-charge has been limiting applications [11]. This can also remove electrons comoving with the protons, without large degradations in beam brightness thereby addressing another issue limiting applications. The most studied laser-based ion beam production process, the target-normal-sheath-acceleration (TNSA) model [11], can achieve proton beams with a broad energy spectrum up to a few dozen $\mathrm{MeV}$ and whose total current is in the $\mathrm{kA}$ range.

Ongoing research based on alternative laser-based ion beam production processes-e.g., radiation pressure 
acceleration, collisionless shock acceleration, breakout afterburner, acceleration in near-critical and underdense plasmas, resistively enhanced acceleration (see Ref. [10]) - offers beams with promising characteristics (e.g., monoenergetic, higher energy and/or higher current) whose space-charge could be successfully controlled by our stack of thin foils.

An idealized analytical envelope theory was previously developed by Lund et al. [6] and agrees with particle-in-cell simulations. This guided an ongoing campaign of experiments, described in Ref. [12] designed to study the mitigation of the defocusing self-electric field of proton beams. These proton beams were produced by intense short-pulse lasers and accelerated by the target-normalshealth-acceleration (TNSA) process [11] at the TITAN laser facility at GSI and the JUPITER laser facility at Lawrence Livermore National Laboratory [13].

This paper extends the idealized analytical envelope theory of Ref. [6], which assumed infinitely thin foils, to include foil-induced scattering and kinetic energy loss associated with finite thickness foils. This scattering causes random deflections in the distribution of particle angles and results in emittance growth that can degrade beam quality $[1,2,14,15]$. The beam ions also deposit a fraction of their kinetic energy into the foils, evaporating the foils after penetration of the beam. The stack of foils is therefore a single-use lens. However, estimates show that the foils remain at near solid density during the transit of the beam within the stack because the hydrodynamical expansion timescale is much longer than the beam transit time scale $[6,12]$. Besides, the ion beam experiences straggling due to statistical kinetic energy losses into the foils, causing momentum spread and therefore chromatic aberrations as the passive focusing depends on the momentum of the beam ions. Straggling is neglected in our present monoenergetic study: the emphasis is on the angular deflections of the beam ions and the mean kinetic energy loss. Nevertheless, straggling must be taken into account when kinetic energy loss is important. Reference [6] includes transverse nonlinear effects between the foils in the envelope equations and indicates that modest changes in the radial structure of the beam associated with geometric aberrations do not significantly alter the passive focusing. Numerical simulations (in which the profile is allowed to evolve self-consistently) show reasonably good agreement with these assumptions.

This paper is organized as follows. In Sec. II, foil-induced scattering is treated analytically using the Rutherford scattering model and numerically using the Monte-Carlo particle simulation code SRIM [16]. In Sec. III, the envelope equations are derived and numerically solved for several foil and beam configurations to highlight cases for which foil-induced scattering becomes a dominant limitation of the transverse focusing. In Sec. IV, a module to model foilinduced scattering and kinetic energy loss is implemented in the particle-in-cell code WARP [17] and is applied to numerically test the envelope theory of Sec. III. Good agreement between the envelope theory and numerical model is found.

\section{SCATTERING}

\section{A. Single particle model}

A single beam ion of velocity $v_{b}$, charge number $Z_{b}$, mass $m_{b}$, and kinetic energy $\mathcal{E}_{b}=\left(\gamma_{b}-1\right) m_{b} c^{2}$, with $\gamma_{b}=\left(1-\beta_{b}^{2}\right)^{-1 / 2}, \beta_{b}=v_{b} / c$, and $c$ the speed of light in vacuum, is assumed to penetrate through a homogenous thin foil of thickness $\Delta_{f}$. The foil is made of a single atomic species of charge number $Z_{f}$, mass $m_{f}$ and mass density $\rho_{f}$. The nuclei and electrons of the foil alter the dynamics of the beam ions differently: the electrons can absorb an appreciable amount of energy from the beam ions without causing significant angular deflections, whereas the nuclei absorb little energy but cause significant angular deflections of the beam ions due to their greater electric charge [18]. In this section, the energy loss of the beam ions due to the collisions with atomic electrons is neglected. This is consistent for thin foils with large incident beam kinetic energy [19]. Small kinetic energy losses within one foil are analyzed in Sec. II B. Results found there justify the constant energy assumption.

Because the interaction between the beam ions and the foil nuclei is primarily electrostatic, the differential scattering cross section $d \sigma / d \Omega$ between the incoming beam ion and a stationary foil nucleus, where the solid angle $d \Omega=\sin \theta d \theta d \phi \quad(\theta$ is the normal angle, taken as the deflection angle, $\phi$ is the azimuthal angle in sphericalpolar coordinates), is governed by the small-angle Rutherford cross-section [18]

$$
\frac{d \sigma}{d \Omega}=\left(\frac{2 Z_{b} Z_{f} e^{2}}{4 \pi \epsilon_{0} m_{e} c^{2}}\right)^{2} \frac{1-\beta_{b}^{2}}{\beta_{b}^{4}} \frac{1}{\theta^{4}} .
$$

Here, $e$ is the elementary electric charge, and $\epsilon_{0}$ the vacuum permittivity. Here and henceforth, large-angle scattering is ignored: those events are rare [18]. Equation (1) is valid between a small cutoff angle

$$
\theta_{\min }=\frac{\hbar}{p_{b} a} \simeq \frac{Z_{f}^{1 / 3}}{192} \frac{m_{e} c}{m_{b} v_{b}}
$$

due to electrostatic screening from bound electrons, and a large cutoff angle

$$
\theta_{\max }=\frac{\hbar}{p_{b} R} \simeq \frac{274}{A_{f}^{1 / 3}} \frac{m_{e} c}{m_{b} v_{b}}
$$

that is due to the finite radius $R$ of the nucleus. In Eqs. (2) and (3), $a \simeq 1.4 a_{0} Z_{f}^{-1 / 3}$ is the length scale of the screening obtained by a rough fit to the Thomas-Fermi atomic 
potential, $a_{0}$ the Bohr radius, $\hbar=h /(2 \pi)$ where $h$ is Planck's constant, $A_{f}$ is the mass number of the nucleus, $m_{e}$ is the mass of the electron, and $p_{b}=m_{b} v_{b}$ is the momentum of a assumed nonrelativistic beam ion. We approximate $A_{f} \simeq 2 Z_{f}$.

The total scattering cross-section is

$$
\sigma_{\mathrm{tot}}=\int_{0}^{2 \pi} d \phi \int_{\theta_{\min }}^{\theta_{\max }} d \theta \sin \theta \frac{d \sigma}{d \theta}
$$

A beam ion traversing a thin foil undergoes many small angle deflections and emerges with a small angular deflection due to the cumulative statistical superposition of many small angle collisions. Assuming the number of collisions is sufficient for Gaussian statistics (verified a posteriori), the central limit theorem applies to the net deflection angle distribution. This implies that the net deflection angle is Gaussian distributed, centered around 0 , with variance $\left\langle\theta^{2}\right\rangle$ given by

$$
\left\langle\theta^{2}\right\rangle \equiv \frac{\int_{0}^{2 \pi} d \phi \int_{\theta_{\min }}^{\theta_{\max }} d \theta \sin \theta \theta^{2} \frac{d \sigma}{d \theta}}{\int_{0}^{2 \pi} d \phi \int_{\theta_{\min }}^{\theta_{\max }} d \theta \sin \theta \frac{d \sigma}{d \theta}} .
$$

The beam ion undergoes $N=n_{f} \sigma_{\text {tot }} \Delta_{f}$ collisions after penetration of a foil of thickness $\Delta_{f}$ and atomic density $n_{f}$. Each of these collisions causes a random deflection $\theta$ that follows the above-mentioned distribution. We take $z$ as the axial coordinate normal to the foil and $x, y$ as the transverse coordinates. Equation (5) corresponds to a deflection $\theta_{x}$ in the $(x-z)$ plane and a deflection $\theta_{y}$ in the $(y-z)$ plane such that $\theta^{2}=\theta_{x}^{2}+\theta_{y}^{2}$ in the small angle approximation (see Fig. 1). By symmetry, the mean and the variance of the total deflection angle at foil exit in the $(x-z)$ and the $(y-z)$ plane are therefore 0 and $\left\langle\theta_{\text {tot }}^{2}\right\rangle \simeq\left\langle\theta_{\mathrm{x}, \text { tot }}^{2}\right\rangle+$ $\left\langle\theta_{\mathrm{y}, \text { tot }}^{2}\right\rangle=2\left\langle\theta_{\mathrm{x}, \text { tot }}^{2}\right\rangle$ because of symmetry, and with $\left\langle\theta_{\mathrm{x}, \text { tot }}^{2}\right\rangle$ and $\left\langle\theta_{\mathrm{y}, \text { tot }}^{2}\right\rangle$ the variance of total deflection angle at foil

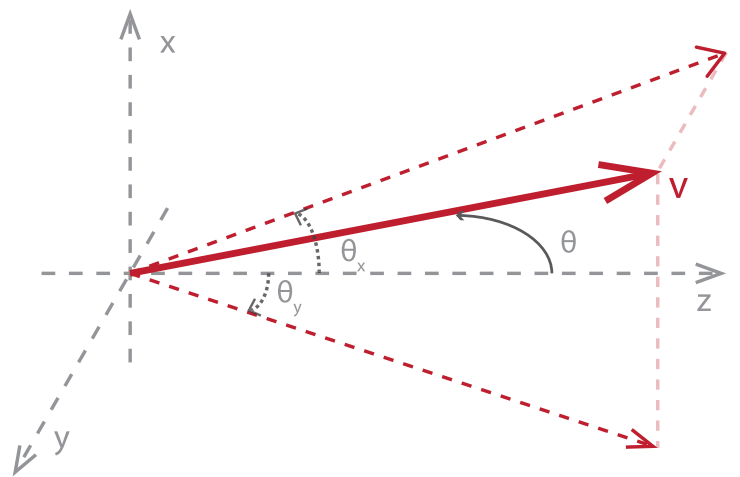

FIG. 1. Schematic of a beam ion at velocity $\mathbf{v}$ that has been deflected by a normal angle $\theta$ from the axial direction $z$. The angular deflections $\theta_{x}$ in the $(x-z)$ plane and $\theta_{y}$ in the $(y-z)$ plane are represented. exit in the $(x-z)$ and $(y-z)$ planes. If $\beta_{b}^{2} \ll 1$, the rms deflection angle in the $(x-z)$ and $(y-z)$ planes reduce to

$$
\begin{gathered}
\left\langle\theta_{\mathrm{x}, \text { tot }}^{2}\right\rangle^{1 / 2}=\left\langle\theta_{\mathrm{y}, \text { tot }}^{2}\right\rangle^{1 / 2}=G_{0} \frac{\Delta_{f}^{1 / 2}}{\mathcal{E}_{b}}, \\
G_{0}=\left[2 \pi n_{f}\left(\frac{Z_{b} Z_{f} e^{2}}{4 \pi \epsilon_{0}}\right)^{2} \ln \left(204 Z_{f}^{-1 / 3}\right)\right]^{1 / 2} .
\end{gathered}
$$

The argument in the logarithm in Eq. (6b) depends on the choice of cutoff angle $\theta_{\min }$ and $\theta_{\max }$ employed which is somewhat arbitrary. However, for our present analysis in which the physics of scattering has been idealized (e.g., electron screening is partially omitted), these specific cutoffs are sufficient. What is of interest here is the scaling of the rms deflection angle distribution $\left\langle\theta_{\mathrm{tot}}^{2}\right\rangle^{1 / 2}$ in Eq. (6a). The scaling is compared and verified by the Monte-Carlo code SRIM in Sec. II B. The code includes a wider range of physical phenomena (more details can be found in Sec. II B). Equation (6a) shows that: (i) higher energy beam ions are less likely to be deflected because of their stiffer trajectories, (ii) higher charge states of the beam ions and higher charged foil nuclei yield broader deflections because the Coulomb interaction is stronger, and (iii) ions undergo larger deflections in denser and thicker foils.

\section{B. Monte-Carlo simulations}

The multiple small-angle scattering of beam ions induced by their penetration through a foil is simulated using the Monte-Carlo code SRIM (stopping and range of ions in matter) [16]. SRIM contains much richer physics than the analytical model used in Sec. II A because it computes the 3D trajectory of a single beam ion through rectangular layers of materials using a quantum mechanical treatment of ion-atom collisions and with adjustments for consistency with experimental data; it also includes screened Coulomb collisions between the beam ion and the foil atoms due to the overlapping electron shells, electron excitations, plasmons, and effective charge $Z_{f}^{*}$ effects where $Z_{f}^{*}<Z_{f}$ due to the collective electron cloud, and large angle scattering. Statistical energy losses, angular scattering, kinetic effects related to energy losses from target damage, sputtering, ionization, and phonon production are also accounted for.

SRIM simulation results for protons with three different initial beam kinetic energies, $\mathcal{E}_{b}=2 \mathrm{MeV}, 5 \mathrm{MeV}$ and $10 \mathrm{MeV}$, penetrating a single foil of thickness $\Delta_{f}$ ranging from $0.125 \mu \mathrm{m}$ to $5 \mu \mathrm{m}$ are shown in Figs. 2 and 3. The material of the foil is solid aluminum $\left(Z_{f}=13\right.$, $\left.\rho=2.7 \mathrm{~g} \mathrm{~cm}^{-3}\right)$. For each initial $\mathcal{E}_{b}$ and $\Delta_{f}, N_{p}=3000$ protons $\left(Z_{b}=1\right)$ are tracked and for each proton $i$, the loss of kinetic energy $\Delta \mathcal{E}_{i}$ and the deflection angle in the transverse direction $\theta_{\mathrm{tot}, i}$ after penetrating the single foil are 


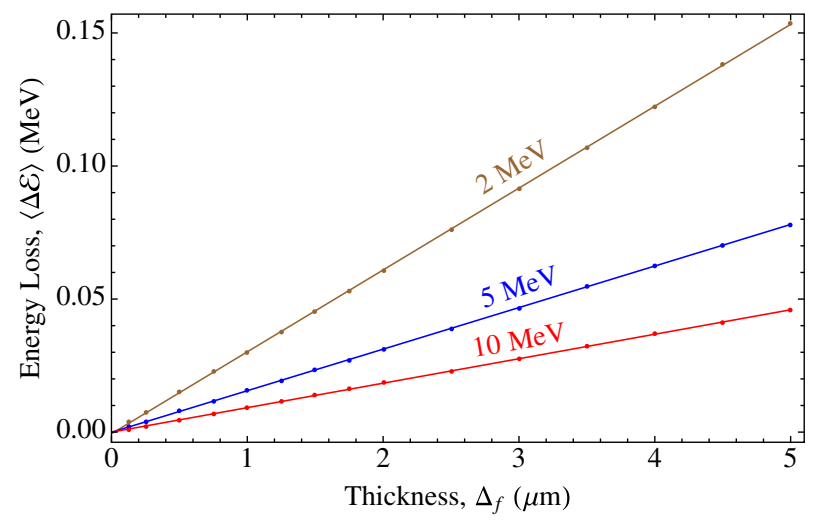

FIG. 2. Dots give smoothed (large angle events rejected) proton kinetic energy loss computed with SRIM, averaged over $N_{p}=$ 3000 protons with initial kinetic energy $\mathcal{E}_{b}$ as indicated after penetration of a solid aluminum foil of thickness $\Delta_{f}$. Smoothing eliminates less than $0.2 \%$ of the simulated protons in the worst case with $\mathcal{E}_{b}=2 \mathrm{MeV}$ and $\Delta_{f}=5 \mu \mathrm{m}$. Solid lines correspond to a linear fit of the data for initial kinetic energies $\mathcal{E}_{b}$. Brown, blue, and red colors represent initial kinetic energies $\mathcal{E}_{b}=2$, 5, and $10 \mathrm{MeV}$.

evaluated. Because of axial symmetry, the deflection angles in both $x$ - and $y$-directions $\theta_{\mathrm{x}, \text { tot }, i}$ and $\theta_{\mathrm{y}, \text { tot }, i}$ are statistically equal. The average of a quantity $A$ is denoted $\langle A\rangle=$ $1 / N_{p} \sum_{i=1}^{N_{p}} A_{i}$ with $A_{i}$ the individual value for the $i$ th proton. SRIM also takes into account rare large angle scatterings, which are not relevant for the bulk of the proton distribution. In our averages, we reject protons whose deflection angle at the exit of the foil is more than 5 standard deviations from the mean. We refer to these averages as "smoothed." We compute, for each initial $\mathcal{E}_{b}$ and $\Delta_{f}$, the average kinetic energy loss $\langle\Delta \mathcal{E}\rangle$, the average

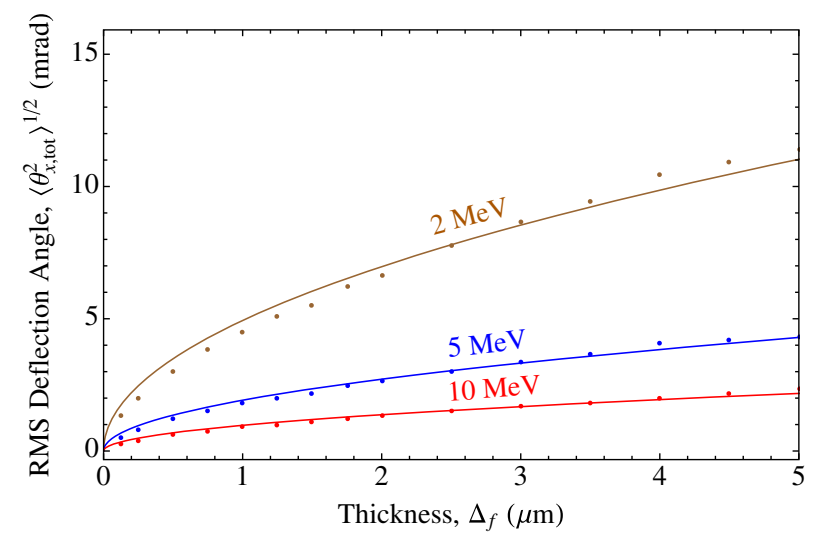

FIG. 3. Dots give smoothed (large angle events rejected) rms deflection angle computed with SRIM, averaged over $N_{P}=3000$ protons with initial ion kinetic energy $\mathcal{E}_{b}$ after penetration of a solid aluminum foil of thickness $\Delta_{f}$. Solid lines correspond to fits of the data based on Eq. (6) using a least-squared method for each initial kinetic energy $\mathcal{E}_{b}$. Brown, blue, and red colors respectively represent the initial kinetic energies of $\mathcal{E}_{b}=2,5$, and $10 \mathrm{MeV}$. transverse angular deflection and average squared transverse angular deflection in the $x$-direction, $\left\langle\theta_{\mathrm{x}, \text { tot }}\right\rangle$ and $\left\langle\theta_{\mathrm{x}, \text { tot }}^{2}\right\rangle$, and in the $y$-direction, $\left\langle\theta_{\mathrm{y}, \text { tot }}\right\rangle$ and $\left\langle\theta_{\mathrm{y}, \text { tot }}^{2}\right\rangle$.

The smoothed average kinetic energy loss $\langle\Delta \mathcal{E}\rangle$, as a function of foil thickness $\Delta_{f}$, is plotted in Fig. 2. As expected, $\langle\Delta \mathcal{E}\rangle$ is small for small $\Delta_{f}$ and high $\mathcal{E}_{b}$. In the case where $\mathcal{E}_{b}=5$ or $10 \mathrm{MeV}$, protons lose a negligible amount of their initial kinetic energy ( $2 \%$ or less), even for foils up to $5 \mu \mathrm{m}$. However, the $2 \mathrm{MeV}$ protons lose close to $8 \%$ of their kinetic energy after penetrating $5 \mu \mathrm{m}$ of solid aluminum. The assumption of constant kinetic energy for beam protons becomes relatively poor for $\mu \mathrm{m}$ thick foils with a proton energy lower than $2 \mathrm{MeV}$. Although the proton kinetic energy can be taken to be constant within one foil, it cannot be assumed to be constant in the full stack of foils because the small decrements in kinetic energy in each foil can result in a substantial net total energy loss when penetrating many foils.

As expected, there is zero mean angular deflection: $\left\langle\theta_{\mathrm{x}, \text { tot }}\right\rangle=\left\langle\theta_{\mathrm{y}, \text { tot }}\right\rangle=0$ (plot not shown). The rms deflection angle $\left\langle\theta_{\mathrm{x}, \text { tot }}^{2}\right\rangle^{1 / 2}\left(=\left\langle\theta_{\mathrm{y}, \text { tot }}^{2}\right\rangle^{1 / 2}\right.$ because of symmetry) as a function of foil thickness $\Delta_{f}$ from the smoothed distribution is plotted in Fig. 3. A least-square fit based on the $\Delta_{f}$ and $\mathcal{E}_{b}$ dependance of Eq. (6) and the results of the MonteCarlo simulations show that

$$
\left\langle\theta_{\mathrm{x}, \text { tot }}^{2}\right\rangle^{1 / 2}=G_{\mathrm{SRIM}} \frac{\Delta_{f}^{1 / 2}}{\mathcal{E}_{b}},
$$

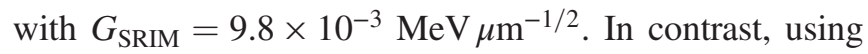
$Z_{b}=1, Z_{f}=13$ and $n_{f}=6.02 \times 10^{28} \mathrm{~m}^{-3}$, the coefficient $G_{0}$ from Eq. (6) gives $G_{0}=2.4 \times 10^{-2}{\mathrm{MeV} \mu \mathrm{m}^{-1 / 2}}^{-1}$ for aluminum which is 2.4 times higher than $G_{\text {SRIM }}$. Such a discrepancy may be justified by the richer models that SRIM employed compared to the model in Sec. II A. Equation (7), is employed in the analysis in the following sections since it should be more accurate. Note also that for $\mathcal{E}_{b}=5 \mathrm{MeV}$ and $10 \mathrm{MeV}$, Eq. (7) produces an excellent fit to the SRIM simulation results. In contrast, data slightly departs from the fit for $2 \mathrm{MeV}$, because the significant loss of kinetic energy for lower energy protons results in enhanced angular scattering.

Methods presented in this section using SRIM can be readily applied to other foil materials and a variety of incident ions.

\section{TRANSVERSE ENVELOPE MODEL}

This section closely follows the treatment in Ref. [6]. First, the beam model, the geometry of the foil system, and the beam fields are described, and then, particle equations of motion both between two foils and within a foil are derived. The particle equations of motion are averaged to obtain an envelope equation for the transverse beam radius. 
Illustrative examples of scattering effects on beam propagation are presented in Sec. III E.

\section{A. Geometry and beam model}

The focusing system (see Fig. 4) is treated as a two-foil system separated by length $L$, perpendicular to the axial $z$ axis, centered at $z=0$ and infinite in the transverse directions $x$ and $y$. This model requires that the characteristic transverse beam radius is much smaller than the transverse extent of the foils. Each foil has finite thickness $\Delta_{f}$ : the beam dynamics is therefore treated differently between the foils and within a foil. The foils are assumed to be grounded conductors.

The ion beam is assumed to be single-species, monoenergetic with kinetic energy $\mathcal{E}_{b}$, mass $m_{b}$ and charge $q=Z_{b} e$, filling the space between many foils. The axial extent of the beam is assumed to be long compared to its transverse size. We describe the beam with a Vlasov model and beam distribution function $f_{b}\left(\mathbf{x}_{\perp}, \mathbf{p}, z\right)$. Because the beam is assumed monoenergetic, the axial coordinate $z$ can be chosen as an independent variable, in place of the time $t$.

The beam charge density $\rho_{b}=q \int d^{3} p f_{b}$ is assumed to be axisymmetric $\left(\partial \rho_{b} / \partial \theta=0\right)$. The $z$-variation of $\rho_{b}$ is neglected between two adjacent foils and within a foil, i.e., $\rho_{b}=\rho_{b}(r)$, where $r=\sqrt{x^{2}+y^{2}}$. However, $\rho_{b}(r)$ is assumed to vary with $\mathrm{z}$ on length scales larger that the interfoil spacing.

The beam line charge $\lambda_{b}=2 \pi \int_{0}^{+\infty} d r r \rho_{b}(r)$ is proportional to the beam current $I_{b}$ with $I_{b}=\beta_{b} c \lambda_{b}$. By definition, the beam current density is $\mathbf{J}_{b}=q \int d^{3} p \mathbf{v} f_{b}$ with $\mathbf{v}$ the particle velocity. The beam is assumed to be mostly axial, $\mathbf{J}_{b} \simeq \hat{\mathbf{z}} \beta_{b} c \rho_{b}(r)$. Here, $\beta_{b} c$ is the axial beam velocity consistent with the axial particle energy $\mathcal{E}_{b}=\left(\gamma_{b}-1\right) m c^{2}$ where $\gamma_{b}=1 / \sqrt{1-\beta_{b}^{2}}$. The radial shape of the charge density $\rho_{b}$ is assumed not to change form throughout the stack of foils (in $z$ ) while the radial extent of the beam charge density is allowed to vary in $z$. This idealization of

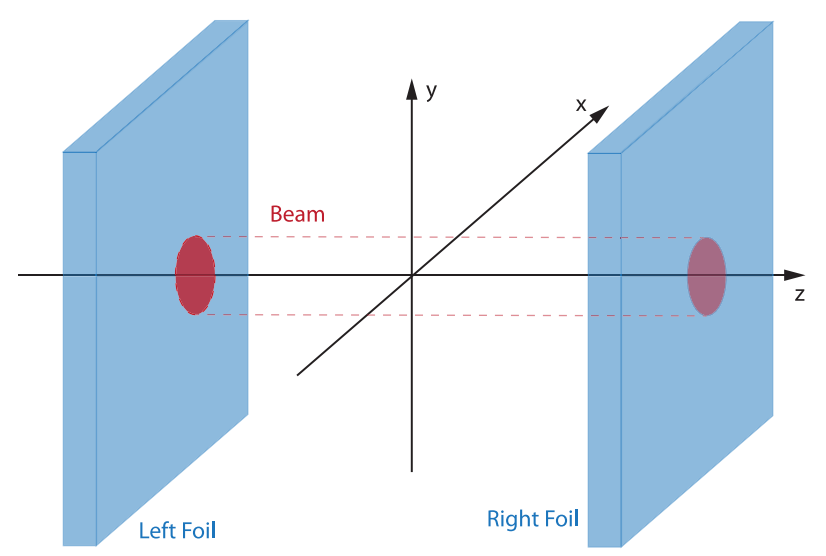

FIG. 4. Axisymmetric beam between two conducting foils located at $z= \pm L / 2$. The foils are grounded. self-similar evolution is consistent with the conservation of the linear charge density $(\lambda=$ const $)$ under radial self-field forces [6].

The transverse $(\perp)$ statistical average of a quantity $A$ over the beam distribution $f_{b}(\mathbf{x}, \mathbf{p}, z)$ is defined by

$$
\langle A\rangle_{\perp} \equiv \frac{\int d^{2} x_{\perp} \int d^{3} p A\left(\mathbf{x}_{\perp}, \mathbf{p}, z\right) f_{b}}{\int d^{2} x_{\perp} \int d^{3} p f_{b}}
$$

where $\mathbf{x}_{\perp}=\hat{\mathbf{x}} x+\hat{\mathbf{y}} y$ denotes the transverse coordinate. The $z$-varying radial extent of the beam charge density is measured by the rms width $\sigma_{x}(z) \equiv\left\langle x^{2}\right\rangle_{\perp}^{1 / 2}$.

\section{B. Self-field solutions}

In this section, the beam electric and magnetic fields are explicitly solved for the case of an axisymmetric beam profile. We employ the quasistatic approximation: $\partial \mathbf{E} / \partial t \simeq 0$ and $\partial \mathbf{B} / \partial t \simeq 0$ in Maxwell's equations. The boundary conditions are set by the conducting foils. $E_{r}$ is screened by the conducting foils: $E_{r}=0$ within the foils, and $B_{\theta}$ remains unmodified by the foils. Details of the derivations can be found in Ref. [6].

\section{Self-magnetic field between two foils}

Using a potential vector $\mathbf{A}$ such that $\mathbf{B}=\boldsymbol{\nabla} \times \mathbf{A}$ and the Coulomb gauge $\boldsymbol{\nabla} \cdot \mathbf{A}=0, \quad \mathbf{A} \simeq \hat{\mathbf{z}} A_{z}$ and $\boldsymbol{\nabla}^{2} A_{z} \simeq$ $-\mu_{0} \beta_{b} c \rho_{b}(r)$. Here, $\mu_{0}$ is the permeability of free space and $c^{2}=1 /\left(\mu_{0} \epsilon_{0}\right)$. $\mathbf{E}$ can be expressed as the gradient of an electrostatic potential in vacuum $\phi_{v}$ such that $\nabla^{2} \phi_{v}=-\rho_{b}(r) / \epsilon_{0}$. The integration of the two previous equations using relevant radial boundary conditions yield $A_{z}(r) \simeq \beta_{b} \phi_{v}(r) / c$. The self-magnetic field has therefore only an azimuthal component

$$
\mathbf{B} \simeq \hat{\boldsymbol{\theta}} B_{\theta}(r) \simeq-\hat{\boldsymbol{\theta}} \frac{\beta_{b}}{\epsilon_{0} c r} \int_{0}^{r} d \tilde{r} \tilde{r} \rho_{b}(\tilde{r})
$$

\section{Self-magnetic field within a foil}

As the thickness of the foils $\Delta_{f}$ is small compared to the interfoil spacing $L\left(\Delta_{f} / L \ll 1\right)$, B is assumed constant and equal to the self-magnetic field at the surface of the foils.

\section{Self-electric field between two foils}

$\mathbf{E}$ can be expressed as the gradient of an electrostatic potential $\phi_{g}$ such that $\mathbf{E}=-\boldsymbol{\nabla} \phi_{g}$ and

$$
\nabla^{2} \phi_{g}=-\frac{\rho_{b}(r)}{\epsilon_{0}} .
$$

In contrast to $\phi_{v}, \phi_{g}$ takes into account the boundary values $\phi_{g}=0$ on the foils at $z= \pm L / 2$. Reference [6] provides the derivation of the solution to Eq. (10): 


$$
\begin{aligned}
\phi_{g}(r, z)= & \frac{1}{\epsilon_{0}} \int_{0}^{\infty} \frac{d k}{k} \frac{\cosh (k L / 2)-\cosh (k z)}{\cosh (k L / 2)} J_{0}(k r) \\
& \times \int_{0}^{\infty} d \tilde{r} \tilde{r} \rho_{b}(\tilde{r}) J_{0}(k \tilde{r}) .
\end{aligned}
$$

Here, $J_{0}$ denotes a 0th order ordinary Bessel function. It was found in Ref. [6] that the radial $E_{r}=-\partial \phi_{g} / \partial r$ and axial $E_{z}=-\partial \phi_{g} / \partial z$ field components could, to a good approximation, be replaced by the $z$-average values between the foils

$$
\begin{aligned}
\bar{E}_{r, g}(r)= & \int_{-L / 2}^{L / 2} \frac{d z}{L} E_{r}(r, z) \\
= & \frac{1}{\epsilon_{0}} \int_{0}^{\infty} d k\left[1-\frac{2}{k L} \tanh (k L / 2)\right] J_{1}(k r) \\
& \times \int_{0}^{\infty} d \tilde{r} \tilde{r} \rho_{b}(\tilde{r}) J_{0}(k \tilde{r}), \\
\bar{E}_{z, g}(r)= & \int_{-L / 2}^{L / 2} \frac{d z}{L} E_{z}(r, z)=0 .
\end{aligned}
$$

\section{Self-electric field within a foil}

The foils are assumed to be perfect conductors so that $\mathbf{E}=0$.

\section{Particle dynamics}

The particle dynamics between the thin foils has been previously treated [6]. This section extends the analysis to include the deleterious effects of scattering within a foil.

The particle dynamics is analyzed in two separate regions: between two foils, which is assumed to be vacuum, and within a perfectly conducting foil. Intrabeam scattering is neglected. Within a foil, deflections of beam ions due to the scattering with foil atoms are included in the equations of motion using the results of Sec. II. A static magnetic field can also be superimposed to improve focusing as treated in Ref. [6].

\section{Between two foils}

The beam charge density is assumed to be axisymmetric, and the foils are assumed to be transversely homogenous, leading to axisymmetric self-fields. The axial self-electric field, $E_{z}(r, z)$, is neglected. In the paraxial approximation $\left(\mathbf{v}=\hat{\mathbf{z}} \beta_{b} c+\delta \mathbf{v} \simeq \hat{\mathbf{z}} \beta_{b} c\right)$, the single particle equation of motion between the foils is

$$
\mathbf{x}_{\perp}^{\prime \prime} \simeq \frac{q}{m \gamma_{b} c^{2}} \frac{\partial \phi_{v}}{\partial \mathbf{x}_{\perp}}-\frac{q}{m \gamma_{b} \beta_{b}^{2} c^{2}} \frac{\partial \phi_{g}}{\partial \mathbf{x}_{\perp}} .
$$

Here, derivatives with respect to $z$ are represented by primes $\left({ }^{\prime}=d / d z\right)$. The first term on the right-hand side of Eq. (13) represents the self-magnetic focusing contribution, and the second term corresponds to the self-electric defocusing contribution.

\section{Within a foil}

Because the foils are assumed to be perfect conductors, no electric field penetrates the foils. The finite thickness of the foils induces Coulomb scattering between beam ions and foil atoms. Therefore, beam ions are both transversely deflected and lose kinetic energy on the foils.

Knock-on electrons emitted from the foils [20] and their effects on the dynamics of the beam ions are a topic for further research. Knock-on electrons fill the gaps between the foils, and the subsequent current neutralization is greater than the subsequent charge neutralization as, by definition, the velocity of the knock-on electrons is higher than the ion beam velocity. Knock-on electrons could therefore mitigate the passive focusing scheme. Nevertheless, the presence of knock-on electrons does not confound the passive focusing in regimes where the foil atoms and the beam ions are of low atomic number, where the beam kinetic energy is high, and where the foils are thin. In this case, the number of generated knock-on electrons would remain negligibly low according to the Rutherford scattering model.

The ion beam kinetic energy $\mathcal{E}_{b}$ is no longer constant and depends on the distance traveled within the foil and the stopping power $\mathcal{S}$ of the foil material. Values of $\mathcal{S}$ are found in tabulated data such as the PSTAR database [21]. Straggling is ignored here, where we consider transverse dynamics. An analysis of straggling would be important for substantial changes in mean beam energy or when energy spread is important. The electric field vanishes in the foil, and terms representing energy loss and the scatteringinduced deflection of the particle are added. The cumulative scattering-induced deflection of a single particle trajectory is a stochastic process that depends on the distance $z$ traveled in the material and the material properties. It is modeled by a Brownian noise $w$ such that for $0 \leq z_{0} \leq z \leq \Delta_{f}, w(z)-w\left(z_{0}\right)$ is a Gaussian distributed variable with mean 0 and variance $\left(z-z_{0}\right) \times G^{2} / \mathcal{E}_{b}^{2}(z)$ to have a form consistent with Eqs. (6) and (7). The instantaneous scattering-induced deflection in the particle equation of motion is therefore represented by the white noise $w^{\prime}$ which is the formal derivative of the Brownian noise $w$. The equation of motion is then the stochastic differential equation

$$
\mathbf{x}_{\perp}^{\prime \prime}+\frac{\left(\gamma_{b} \beta_{b}\right)^{\prime}}{\left(\gamma_{b} \beta_{b}\right)} \mathbf{x}_{\perp}^{\prime}-\frac{q}{m \gamma_{b} c^{2}} \frac{\partial \phi_{v}}{\partial \mathbf{x}_{\perp}} \simeq w^{\prime},
$$

and includes the particle kinetic energy loss due to the stopping power of the foil material $\mathcal{E}_{b}^{\prime}=S\left(\mathcal{E}_{b}\right)$ (see Fig. 5), or equivalently

$$
\left(\gamma_{b} \beta_{b}\right)^{\prime}=\frac{S\left(\mathcal{E}_{b}\right)}{m c^{2} \beta_{b}} .
$$




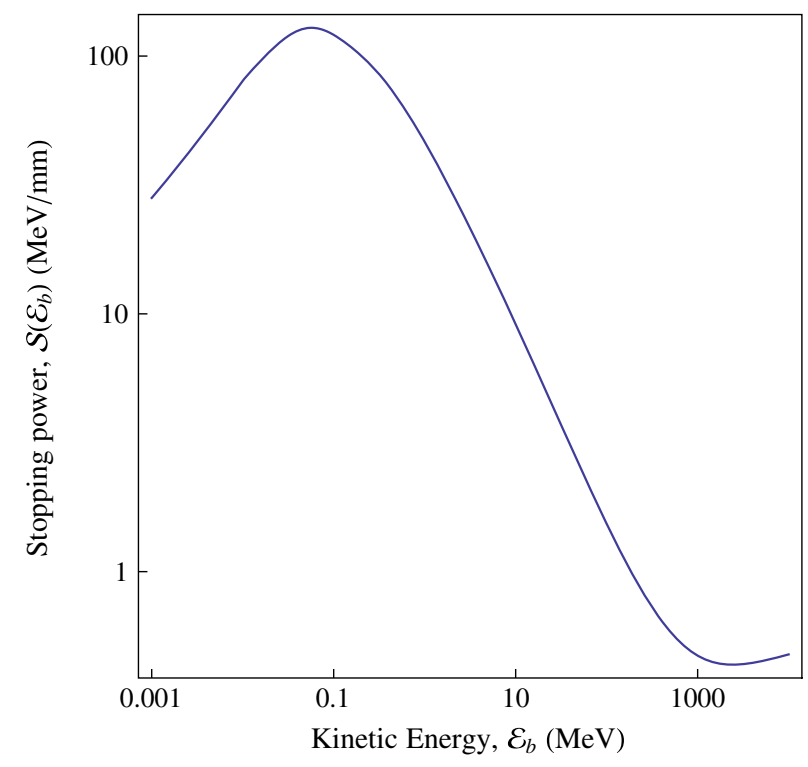

FIG. 5. Stopping power $\mathcal{S}\left(\mathcal{E}_{b}\right)$ of a proton in solid aluminum (mass density $\rho=2.7 \mathrm{~g} / \mathrm{cm}^{3}$ ).

The deceleration-induced term $\frac{\left(\gamma_{b} \beta_{b}\right)^{\prime}}{\left(\gamma_{b} \beta_{b}\right)} \mathbf{x}_{\perp}^{\prime}$ is derived in Ref. [2].

\section{Envelope equations}

The statistical transverse envelope equations are derived by taking the derivatives in $z$ of $\sigma_{x}(z)$ and the transverse rms emittance of the beam $\varepsilon_{x, \text { rms }} \equiv\left[\left\langle x^{2}\right\rangle_{\perp}\left\langle x^{\prime 2}\right\rangle_{\perp}-\right.$ $\left.\left\langle x x^{\prime}\right\rangle_{\perp}^{2}\right]^{1 / 2}$, and then applying the particle equations between two foils and within one foil. Because the beam is axisymmetric, only the $x$-component of the beam envelope equation is treated. This section extends the envelope formalism of Ref. [6] to include additional effects due to scattering within a foil.

\section{Between two foils}

The beam envelope equation between two foils, derived in Ref. [6], is

$$
\frac{d^{2}}{d z^{2}} \sigma_{x}+\frac{\gamma_{b}^{2}}{4}\left[\beta_{b}^{2}-\bar{F}\right] \frac{Q}{\sigma_{x}}-\frac{\varepsilon_{x, \mathrm{rms}}^{2}}{\sigma_{x}^{3}}=0 .
$$

The dimensionless perveance $Q=q \lambda_{b} /\left(2 \pi \epsilon_{0} m \gamma_{b}^{3} \beta_{b}^{2} c^{2}\right)$ is constant $[1,2,22]$. It is assumed that, between the foils, the nonlinear field effects are small and therefore $\varepsilon_{x, \text { rms }}$ is constant.

$$
\bar{F}=-\frac{4 \pi \epsilon_{0}}{\lambda}\left\langle r \int_{-L / 2}^{L / 2} \frac{d z}{L} \frac{\partial \phi_{g}}{\partial r}\right\rangle_{\perp}
$$

is a dimensionless "form factor" that models the average screening of the defocusing field due to the foils for closely spaced foils $\left(L \ll \rho(\partial \rho / \partial z)^{-1}\right)$ as the beam ions cannot rapidly respond to fast variations of the defocusing electric field between closely space foils (see Ref. [6] for details). Scattering does not change this result as it does not happen between the foils. In vacuum, $\bar{F}=1$ and the envelope equation reduces to the familiar vacuum form [6]. The form factor $\bar{F} \in[0,1]$ can be effectively seen as an attenuation factor of the defocusing electric field due to the foils.

\section{Within a single foil}

In this paragraph, $z=0$ is taken at the middle of the foil and the foil domain in $z$ is $\left[-\Delta_{f} / 2, \Delta_{f} / 2\right]$. Equation (14) and the derivatives of $\left\langle x^{2}\right\rangle_{\perp}^{1 / 2}$ in $z$ yield the beam envelope equation within a single foil.

$$
\frac{d^{2}}{d z^{2}} \sigma_{x}+\frac{\left(\gamma_{b} \beta_{b}\right)^{\prime}}{\left(\gamma_{b} \beta_{b}\right)} \frac{d}{d z}\left\langle x x^{\prime}\right\rangle_{\perp}^{1 / 2}+\frac{\gamma_{b}^{2}}{4} \beta_{b}^{2} \frac{Q}{\sigma_{x}}-\frac{\varepsilon_{x, \mathrm{rms}}^{2}}{\sigma_{x}^{3}}=\frac{\left\langle x w^{\prime}\right\rangle_{\perp}}{\sigma_{x}} .
$$

Equation (18) differs from the beam envelope equation between two foils, Eq. (16), by the absence of a defocusing electric field, and the presence of scattering and deceleration. Furthermore, the emittance is not conserved because of both kinetic energy losses and cross-terms between $x, x^{\prime}$ and $w^{\prime}$ due to scattering-induced deflections. Differentiating $\varepsilon_{x, \text { rms }}^{2}$ with respect to $z$ and applying Eq. (14) yields

$\frac{d}{d z} \varepsilon_{x, \mathrm{rms}}^{2}=-2 \frac{\left(\gamma_{b} \beta_{b}\right)^{\prime}}{\gamma_{b} \beta_{b}} \varepsilon_{x, \mathrm{rms}}^{2}+2 \sigma_{x}^{2}\left\langle x^{\prime} w^{\prime}\right\rangle_{\perp}-2\left\langle x x^{\prime}\right\rangle_{\perp}\left\langle x w^{\prime}\right\rangle_{\perp}$.

In Appendix A, a detailed analysis of the cross-terms for beam kinetic energy $\mathcal{E}_{b}$ leads to Eqs. (A10) and (A13), and shows that $\left\langle x w^{\prime}\right\rangle_{\perp}=0$ and $\left\langle x^{\prime} w^{\prime}\right\rangle_{\perp}=G^{2} /\left(2 \mathcal{E}_{b}^{2}\right)$. Using these results, Eq. (18) reduces to

$\frac{d^{2}}{d z^{2}} \sigma_{x}+\frac{\left(\gamma_{b} \beta_{b}\right)^{\prime}}{\gamma_{b} \beta_{b}} \frac{d}{d z}\left\langle x x^{\prime}\right\rangle_{\perp}^{1 / 2}+\frac{\gamma_{b}^{2}}{4} \beta_{b}^{2} \frac{Q}{\sigma_{x}}-\frac{\varepsilon_{x, \mathrm{rms}}^{2}}{\sigma_{x}^{3}}=0$,

and Eq. (19) reduces to

$$
\frac{d}{d z} \varepsilon_{x, \mathrm{rms}}^{2}=-2 \frac{\left(\gamma_{b} \beta_{b}\right)^{\prime}}{\gamma_{b} \beta_{b}} \varepsilon_{x, \mathrm{rms}}^{2}+\frac{G^{2}}{\mathcal{E}_{b}^{2}} \sigma_{x}^{2} .
$$

For a paraxial beam, angles of particle trajectories in the foil are small and the total distance traveled by the ions within the foil is, therefore, to first approximation, $\Delta_{f}$. The beam size can be approximated as constant within an individual foil $\left(\sigma_{x}=\right.$ const) when the foils are thin compared to transverse focal length. Then, the kinetic energy loss $\Delta \mathcal{E}_{b}$ of the ion beam reduces to

$$
\Delta \mathcal{E}_{b} \simeq \mathcal{S}\left(\mathcal{E}_{b}\left(-\Delta_{f} / 2\right)\right) \Delta_{f} \simeq \mathcal{S}\left(\mathcal{E}_{b}\right) \Delta_{f} .
$$

Generally, $\left|\Delta \mathcal{E}_{b}\right| \ll \mathcal{E}_{b}$, and the energy $\mathcal{E}_{b}$ can be assumed constant within a single foil when computing quantities that 
are functions of $\mathcal{E}_{b}$ because the higher-order induced errors are small. The emittance evolution equation (19) can then be integrated across a foil,

$$
\begin{aligned}
\varepsilon_{x, \mathrm{rms}}^{2}\left(\Delta_{f} / 2\right)= & \varepsilon_{x, \mathrm{rms}}^{2}\left(-\Delta_{f} / 2\right)+\int_{-\Delta_{f} / 2}^{\Delta_{f} / 2} d z \frac{d}{d z} \varepsilon_{x, \mathrm{rms}}^{2}(z) \\
\simeq & \varepsilon_{x, \mathrm{rms}}^{2}\left(-\Delta_{f} / 2\right) \\
& +\Delta_{f}\left[-2 \frac{\left(\gamma_{b} \beta_{b}\right)^{\prime}}{\gamma_{b} \beta_{b}} \varepsilon_{x, \mathrm{rms}}^{2}+\frac{G^{2}}{\mathcal{E}_{b}^{2}} \sigma_{x}^{2}\right]_{z=-\Delta_{f} / 2} .
\end{aligned}
$$

In Eq. (23), terms of order $\Delta_{f}^{2}$ and higher are neglected. Because the foils induce deceleration of the ions, $\left(\gamma_{b} \beta_{b}\right)^{\prime}$ is negative, causing emittance growth. Because $G$ is always positive, the scattering term also causes emittance growth. Note that while the kinetic energy loss within one foil is small compared to the kinetic energy of the beam, the accumulated losses of kinetic energy due to its propagation through a large number of foils can be significant and should be accounted for in the beam dynamics. We employ the thin foil approximation and apply Eqs. (22) and (23) in the following sections.

Note that, instead of our previously defined transverse emittance, it is possible to use the normalize transverse emittance as a measure of beam quality as it is a conserved quantity under acceleration or deceleration. In this case, an auxillary equation for $\gamma_{b}^{\prime}$ [2] must be taken into account.

\section{E. Example: Application of the envelope model to intense proton beams}

We analyze a lens where thin foils of constant thickness $\Delta_{f}$ are stacked with constant foil spacing $L$. Modulation of foil spacing $L$ as a function of the beam radial size can optimize the focusing mechanism, but is not treated here. The foil material is solid aluminum $\left(\rho=2.7 \mathrm{~g} \mathrm{~cm}^{-3}\right.$, $Z_{f}=13$ ), with angular deflection coefficient $G_{\text {SRIM }}=$ $9.8 \times 10^{-3} \mathrm{MeV} \mu \mathrm{m}^{-1 / 2}$ from Sec. II and the stopping power $\mathcal{S}\left(\mathcal{E}_{b}\right)$ extracted from Ref. [21]. Use of conducting materials different from solid aluminum results in a different deflection coefficient $G$ that can be recomputed using the methods of Sec. II, and a different stopping power $\mathcal{S}\left(\mathcal{E}_{b}\right)$. The continuous approximation of the form factor $\bar{F}$ from Eq. (17) is used. No external focusing system is employed. The ion beam is assumed to have no angular momentum. Secondary electrons and neutralizing plasma are neglected.

For efficient passive focusing, the beam must ideally be high current and high energy consistently as analyzed in Sec. III D. For example, consider a proton beam with a high perveance value of $Q=1.8 \times 10^{-2}-\mathrm{e}$.g., a monoenergetic $4.8 \mathrm{kA} 30 \mathrm{MeV}$ proton beam. Such characteristics may be achievable in the near future by laser-produced proton beams as the individual characteristics can already be separately reached [10]. The initial beam density is radially Gaussian with rms beam width $\sigma_{x}=200 \mu \mathrm{m}$ and zero divergence $\sigma_{x}^{\prime}=0$. The foil spacing is set to $L=100 \mu \mathrm{m}$ and the foil thicknesses $\Delta_{f}$ range from 0 to $12.8 \mu \mathrm{m}$. The initial beam emittance is $\varepsilon_{x, \mathrm{rms}}=0.87 \mathrm{~mm}$ mrad. The emittance in this case grows due to foil-induced scattering in the thin, but finite thickness foils. The finite foil thickness induces a reduction of focusing that we quantify by the focusing efficiency defined by

$$
\eta\left(\Delta_{f}\right)=\frac{\sigma_{\mathrm{x}, \text { init }}-\sigma_{\mathrm{x}, \min }\left(\Delta_{f}\right)}{\sigma_{\mathrm{x}, \text { init }}-\sigma_{\mathrm{x}, \min }\left(\Delta_{f}=0\right)} .
$$

Here, $\sigma_{\mathrm{x}, \text { init }}$ is the initial rms beam width. $\sigma_{\mathrm{x}, \min }\left(\Delta_{f}\right)$ is the best focus for foils with thickness $\Delta_{f}$, occurring at a distance $z_{\min }$ after the first foil. $z_{\min }$ is called the effective focal length. The minimum beam rms width for infinitely thin foils is $\sigma_{\mathrm{x}, \min }\left(\Delta_{f}=0\right)$. This definition of the focusing efficiency factor $\eta$ is valid only when the foil spacing is small enough to induce initial focusing. The focusing efficiency $\eta$ is desired to be as close as possible to unity, corresponding to small defocusing degradation due to scattering and energy losses. Mitigation of foil-induced scattering can be achieved by reducing the foil thickness $\Delta_{f}$, the addition of an external focusing system, or using higher initial beam energy $\mathcal{E}_{b}$.

The dependence of the focusing efficiency $\eta$ as a function of foil thickness $\Delta_{f}$ for various initial proton kinetic energies is shown in Fig. 6 . The beam rms width $\sigma_{x}$, emittance and energy for various foil thicknesses and an initial beam kinetic energy $\mathcal{E}_{b}=30 \mathrm{MeV}$ are plotted in Fig. 7. The plot of the beam rms width shows that, as expected, thicker foils decrease the maximum beam focus, but are still preferable to the vacuum case where the beam quickly expends. The plot of the axial beam kinetic energy $\mathcal{E}_{b}$ is consistent with the stopping power of the employed tabulated PSTAR data in Ref. [21]. Emittance growth is observed in the plot of the beam emittance. Table I

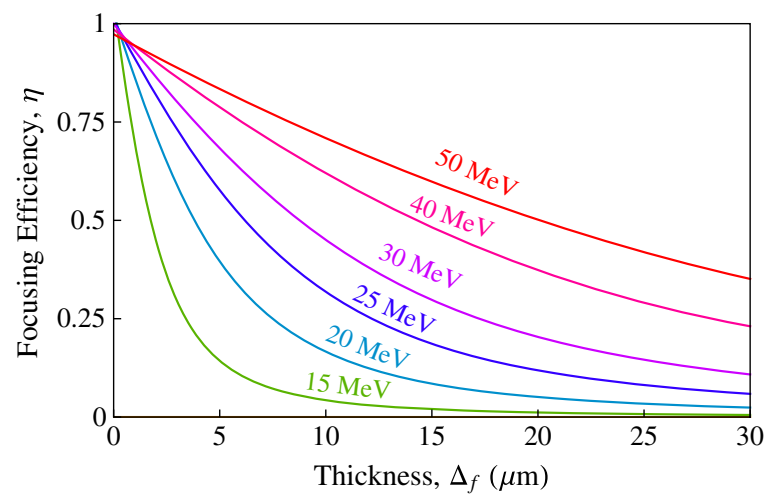

FIG. 6. Proton focusing efficiency $\eta$ as a function of foil thickness $\Delta_{f}$ is computed for specified initial kinetic energies $\mathcal{E}_{b}$. Green, cyan, blue, purple, pink, red colors represent initial kinetic energies $\mathcal{E}_{b}=15,20,25,30,40$, and $50 \mathrm{MeV}$. 

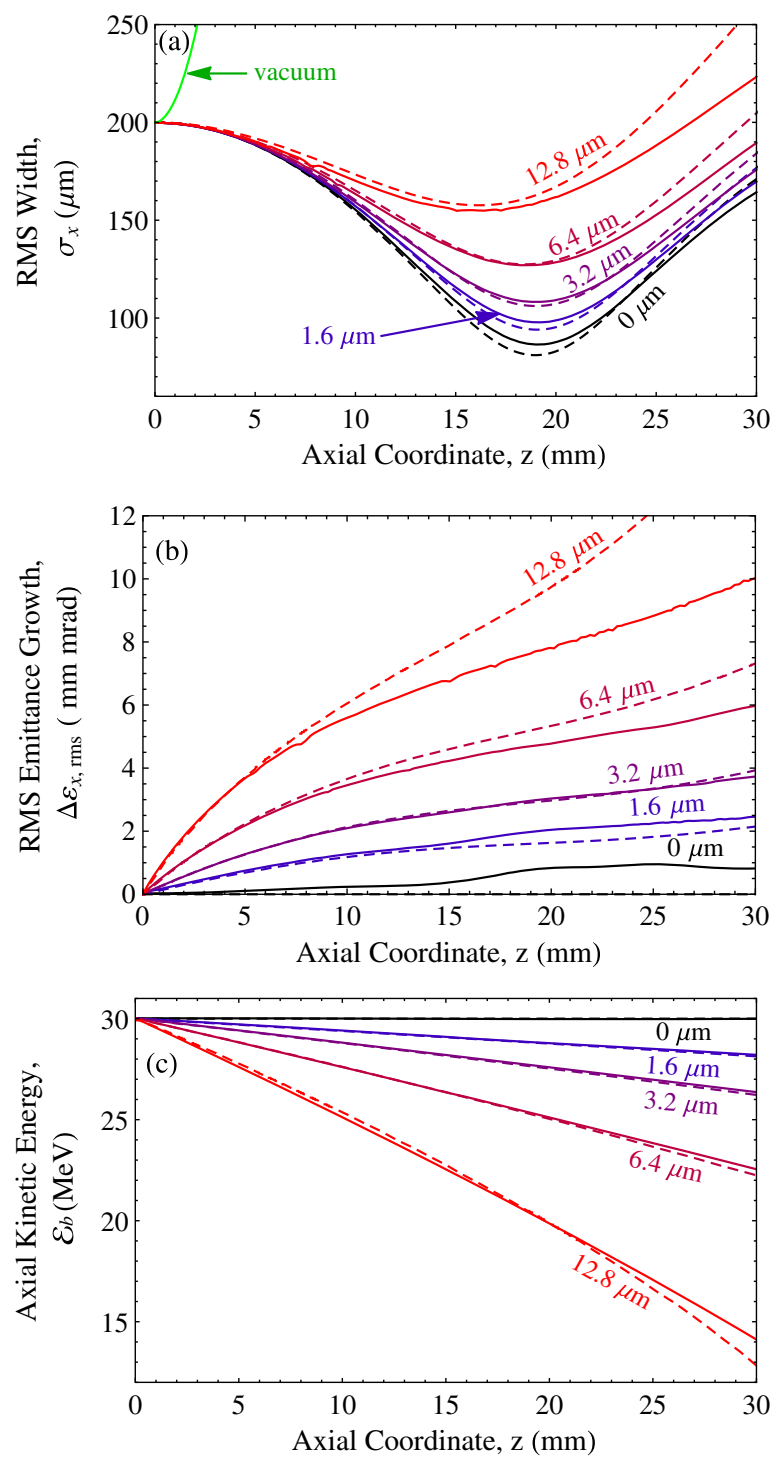

FIG. 7. The evolution of rms beam width $\sigma_{x}$ (a), rms transverse emittance growth $\Delta \varepsilon_{x, \text { rms }}$ (b), and axial kinetic energy $\mathcal{E}_{b}$ (c) as a function of $z$ for foil spacing $L=100 \mu \mathrm{m}$ and foil thickness $\Delta_{f}=0,1.6,3.2,6.4,12.8 \mu \mathrm{m}$ as labeled. Quantities at the focal spot ( $z$ position of the smallest $\sigma_{x}$ ) are summarized in Table. I. Dashed lines represent the envelope model solutions. Solid lines represent WARP simulations.

summarizes the beam size and emittance at maximum focusing for a variety of foil thicknesses. Plots of particlein-cell simulation results obtained from WARP [17] (see Sec. IV) are also included in Fig. 7. The focusing efficiency is quickly reduced with thicker foils, which moves the focal spot closer to the entrance of the stack of the thin foils, reduces the beam kinetic energy, and increases the beam emittance. The effects are more deleterious for a beam with lower perveance (i.e., less magnetic focusing) and lower energy (i.e., more scattering and faster kinetic energy loss). For example, a cold $10 \mathrm{MeV}$ proton beam of perveance $8.14 \times 10^{-3}$ would have a focusing efficiency of $9 \%$ for
TABLE I. Minimum $\sigma_{\mathrm{x}, \min }$ and corresponding $z$-location $z_{\min }$ (effective focal length) for foil spacing $L=100 \mu \mathrm{m}$ and initial kinetic energy $\mathcal{E}_{b}=30 \mathrm{MeV}$ for different foil thicknesses $\Delta_{f}$. Corresponding focusing efficiency $\eta$, kinetic energy $\mathcal{E}_{b}$ at $\sigma_{\mathrm{x}, \min }$, and beam rms emittance growth $\Delta \varepsilon_{x, \mathrm{rms}}$ at $\sigma_{\mathrm{x}, \mathrm{min}}$ are displayed for the $30 \mathrm{MeV}$ initial beam shown in Fig. 6 and 7.

\begin{tabular}{|c|c|c|c|c|c|}
\hline \multirow{3}{*}{$\begin{array}{l}\text { Foil } \\
\text { Thickness } \\
\Delta_{f}(\mu \mathrm{m})\end{array}$} & \multicolumn{5}{|c|}{ Foil Spacing } \\
\hline & \multicolumn{5}{|c|}{$L=100 \mu \mathrm{m}$} \\
\hline & $\sigma_{\mathrm{x}, \min }(\mu \mathrm{m})$ & $\eta$ & $\begin{array}{c}z_{\min } \\
(\mathrm{mm})\end{array}$ & $\begin{array}{c}\mathcal{E}_{b} \\
(\mathrm{MeV}) \\
\end{array}$ & $\begin{array}{c}\Delta \varepsilon_{x, \mathrm{rms}} \\
(\mathrm{mm} \mathrm{mrad})\end{array}$ \\
\hline 0 & 81.0 & 1 & 19.0 & 30 & 0 \\
\hline 1.6 & 94.1 & 0.89 & 19.1 & 28.8 & 0.4 \\
\hline 3.2 & 106.1 & 0.79 & 19.0 & 27.7 & 0.7 \\
\hline 6.4 & 127.5 & 0.61 & 18.4 & 25.4 & 1.2 \\
\hline 12.8 & 157.6 & 0.36 & 16.1 & 22.1 & 2.1 \\
\hline
\end{tabular}

even an extremely thin foil of thickness $\Delta_{f}=160 \mathrm{~nm}$, which means that passive focusing cannot effectively operate for such low energy and low perveance beams. In order to achieve a focusing efficiency of $70 \%$ for such a beam, the foil thickness would have to be about $24 \mathrm{~nm}$. Nonetheless, even though focusing cannot be achieved, the stack of foils strongly mitigates defocusing compared to vacuum values (see Fig. 7). Results presented here help clarify where idealized results from Ref. [6], in which scattering and energy losses were neglected, can be reliably applied.

Since passive focusing is nonlinear (the focusing term in the envelope equation is proportional to $Q / \sigma_{x}$ in contrast to solenoidal focusing that is linear, i.e., proportional to $\kappa \sigma_{x}$ where $\kappa$ is the applied focusing function), equivalence in terms of thin lens optics is not possible. Therefore, as an approximate comparison between passive focusing and solenoidal focusing, we compute the necessary solenoidal magnetic field to reach the same minimum spot size $\sigma_{\mathrm{x}, \mathrm{min}}$ provided by passive focusing. For the above-mentioned beam parameters, in the absence of foils, a solenoidal magnetic field of $600 \mathrm{~T}$ would be required to reach the minimum spot size $\sigma_{\mathrm{x}, \min }=157.6 \mu \mathrm{m}$ that is provided by a stack of thin foils of thickness $\Delta_{f}=12.8 \mu \mathrm{m}$. This shows the advantage of foil focusing relative to vacuum focusing with applied fields for the beam parameters examined here. In optimized systems, it may be advantageous to use combined solenoid and foil focusing, using fewer foils and the solenoid strength where the beam is large, and more foils as the beam focuses. This could partly mitigate scattering issues and give more system tunability. Note that, while quadrupoles are also linear optics, their uses are more even problematic. One could indeed superimpose high gradient pulsed magnet quadrupole periodic lattices to replace the solenoid focusing. Estimates show that for an occupancy of 0.5 and the quadrupole length $L=2 \mathrm{~mm}$ (this short length is required for the effective focal length $z_{\min }$ to be in the same range as the study above, i.e., in the 
tens of $\mathrm{mm}$ range), the required magnetic gradient is $6 \times 10^{5} \mathrm{~T} / \mathrm{m}$, or, equivalently, a field of $30 \mathrm{~T}$ for a radius of $50 \mu \mathrm{m}$. These are extreme fields. Alternatively, to avoid those extreme fields, one could use upstream (nonimmersed) quadrupole optics in a combined type final focus using permanent magnets, but that also introduces another issue regarding the behavior of electrons respective to the dynamics of ions when entering the quadrupoles.

\section{SIMULATIONS}

The envelope model is compared to particle-in-cell simulations using the WARP code [17] in axisymmetric cylindrical $(r-z)$ geometry with a regular grid. The basic simulation model is also discussed in Ref. [6]; it is generalized here to include scattering and energy loss effects. The setup of the present simulations is similar to what was referred as the "infinite beam" simulation setup of Ref. [6], and agrees well with its analytical envelope model that excludes scattering and energy loss effects. The domain is bounded radially by the beam pipe at $r=$ $1.2 \mathrm{~mm}$ and axially by the ends of two adjacent foils, and contains by 64 radial grid cells and 8 axial grid cells. The boundary conditions for macroparticles are absorbing in the $r$ direction and periodic in the $z$ direction. This choice of boundary conditions for particles speeds up the simulation as particles exiting from the right end are reinjected back into the domain from the left end with the same velocity and the same transverse position. This bypasses the need for much larger simulations of the whole stack of thin foils and focuses on the beam dynamics between two foils and within one foil. The electric field is calculated in the electrostatic approximation with Dirichlet boundary conditions in $r$ and in $z$. The magnetic field is calculated in the magnetostatic approximation with Dirichlet boundary conditions in $r$ and periodic boundary conditions in $z$. Beam macroparticles are initially loaded with regular spacing in the $z$ direction.

In the $r$ direction, the particles are loaded following an initially axisymmetric Gaussian charge profile, chopped at $r=r_{\max }=3.5 \sigma_{b}$. The particles are spaced uniform in $R^{2}$ out to $R=r_{b}=2 \sigma_{b}$, with $R$ related to the actual radius $r$ by the relation

$$
r=\sigma_{x} \sqrt{-2 \ln \left[1-\left(\frac{R}{2 r_{b}}\right)^{2} \mathcal{N}\right]} .
$$

with $\mathcal{N}=1-\exp \left(-r_{\max }^{2} /\left(2 \sigma_{b}^{2}\right)\right)$, a normalization factor due to the chopping (see Appendix B for details).

The rms transverse beam size $\sigma_{x}$ is computed by averaging over the full axial domain. Typically, 296 macroparticles are loaded per particle-containing cell. These simulated beam parameters are identical to the beam parameters of the envelope model of Sec. III E for direct comparison. Parametric numerical studies in the absence of scattering showed that the grid resolution and statistics were sufficient for well converged simulations [6]. Scattering and energy losses are not expected to change requirements.

Scattering and energy loss options were added to the modeling of foils in WARP. Foils are located at each axial end of the domain and assumed to extend to the radial boundaries. When a particle penetrate a foil, the particle is given a random transverse kick that follows the normal distribution with mean 0 and variance $\left\langle\theta_{\mathrm{x}}^{2}\right\rangle=G_{\mathrm{SRIM}}^{2} \Delta_{f} / \mathcal{E}_{b}^{2}$ [see Eq. (7)]. After scattering, the kinetic energy of the beam $\mathcal{E}_{b}$ is reduced by $\Delta \mathcal{E}_{b} \simeq \mathcal{S}\left(\mathcal{E}_{b}\left(-\Delta_{f} / 2\right)\right) \Delta_{f}$ [see Eq. (22)].

Results of these simulations are shown in Fig. 7 and agree reasonably well with the envelope results for the axial kinetic energy $\mathcal{E}_{b}$. The minor discrepancies between the simulations and the envelope model are due to various effects not included in the envelope model as mentioned in Ref. [6]: (i) the radial density is evolving and does not stay Gaussian, (ii) the electric field is not averaged between the foil by using $\bar{F}$, and (iii) emittance growth due to the nonlinear nature of the self-fields. Thicker foils enhance these differences.

\section{REMARKS}

This paper generalized recent theory and simulation models in Ref. [6] to include the degradation of beam quality due to foil-induced scattering and energy loss in passive focusing. This study shows that a higher beam kinetic energy, a lower beam atomic number and/or foil thickness are needed for optimal passive focusing using a stack of thin foils. Extending the study to a larger range of foil properties (e.g., with irregular spacing and thickness, different shape, holes) would open possibilities to optimized passive focusing systems for more complex beams (e.g., with large energy spectrum, not initially collimated, with comoving and secondary elections).

\section{ACKNOWLEDGMENTS}

This work was performed under the auspices of the U.S. Department of Energy by Lawrence Livermore National Security, LLC, Grant No. DE-AC52-07NA27344, and supported at UC Berkeley under Grant No. DE-FG0204ER41289.

\section{APPENDIX A: CALCULATION OF THE MOMENTS $\left\langle\boldsymbol{x} \boldsymbol{w}^{\prime}\right\rangle$ AND $\left\langle\boldsymbol{x}^{\prime} \boldsymbol{w}^{\prime}\right\rangle$ WITHIN METALLIC FOILS}

Consider Eq. (14) with constant kinetic energy $\left(\gamma_{b} \beta_{b}=\right.$ const $)$ and in the $x$ direction. By introducing $K=-\frac{q}{m \gamma_{b} c^{2} x} \frac{\partial \phi_{v}}{\partial x}$, a Hill's equation with a stochastic term is obtained,

$$
x^{\prime \prime}(z)+K(z) x(z)=w^{\prime}(z)
$$


where $w^{\prime}$ is white noise that we model as a sum of discrete kicks with

$$
w^{\prime}(z)=\sum_{i=1}^{n} \Delta_{i} \delta\left(z-z_{i}\right)
$$

Here, $\delta(z)$ is the Dirac delta function, $z_{i}$ is the axial position where the $i$ th transverse kick occurs, $n$ is the total number of transverse kicks from the axial coordinate $-\Delta_{f} / 2$ to $z$, and $\Delta_{i}$ the amplitude of the $i$ th kick. From Sec. II B, the kicks $\Delta_{i}$ are normal distributed centered on 0 with variance $G^{2} / \mathcal{E}_{b}^{2}(z) \delta z$. Here $\delta z$ is the mean free path between two collisions. Between two kicks (i.e., for any $z \neq z_{i}$ with $i \in \llbracket 1, n \rrbracket)$, Eq. (A1) reduces to the regular Hill's equation,

$$
x^{\prime \prime}(z)+K(z) x(z)=0
$$

The solution of the regular Hill's equation, Eq. (A3), between the $i$ th kick and the $(i+1)$ th kick has the form

$$
\begin{aligned}
x_{i}(z)= & {\left[A_{i} f_{i}\left(z-z_{i}\right)+B_{i} g_{i}\left(z-z_{i}\right)\right]\left[H\left(z-z_{i}\right)\right.} \\
& \left.-H\left(z-z_{i+1}\right)\right] .
\end{aligned}
$$

Here, $A_{i}$ and $B_{i}$ are constants that depend on the initial conditions, $C_{i}$ and $S_{i}$ are cosine-like and sine-like functions satisfying Eq. (A3) with initial conditions $C_{i}(0)=1$, $C_{i}{ }^{\prime}(0)=0, \quad S_{i}(0)=0, S_{i}{ }^{\prime}(0)=1$, and $H$ is a "step" function defined such that

$$
H(z)= \begin{cases}1, & z>0 \\ \frac{1}{2}, & z=0 \\ 0, & z<0\end{cases}
$$

The general solution of Eq. (A1) can be expressed as

$$
x(z)=\sum_{i=0}^{n} x_{i}(z)
$$

Consider a particle with initial conditions $x\left(z_{0}\right)=x_{0}$ and $x^{\prime}\left(z_{0}\right)=x_{0}^{\prime}$ where $z_{0}=-\Delta_{f} / 2$. This sets $A_{0}=x_{0}$, $B_{0}=x_{0}^{\prime}$. Note that $z_{i}>z_{i-1}$ for $i>0$. Then, the following equations recursively hold for any $i>0$ :

$$
\begin{aligned}
& A_{i}=A_{i-1} C_{i-1}\left(z_{i}-z_{i-1}\right)+B_{i-1} S_{i-1}\left(z_{i}-z_{i-1}\right), \\
& B_{i}=A_{i-1} C_{i-1}^{\prime}\left(z_{i}-z_{i-1}\right)+B_{i-1} S_{i-1}^{\prime}\left(z_{i}-z_{i-1}\right)+\Delta_{i} .
\end{aligned}
$$

It can be shown that, for any $i>1$,

$$
A_{i}=\mathcal{A}_{i}+\sum_{j=1}^{i-1} \mathcal{C}_{i, j} \Delta_{j}, B_{i}=\mathcal{B}_{i}+\sum_{j=1}^{i-1} \mathcal{D}_{i, j} \Delta_{j}+\Delta_{i}
$$

where $\mathcal{A}_{i}, \mathcal{B}_{i}, \mathcal{C}_{i, j}, \mathcal{D}_{i, j}$ are constants that depend solely on $A_{0}, B_{0}$, and $C_{i}, C_{i}{ }^{\prime}, S_{i}, S_{i}{ }^{\prime}$ evaluated at $z_{i}$ and $z_{i-1}$. Their explicit evaluation is not necessary in our analysis.

We can now compute $\left\langle x w^{\prime}\right\rangle$ and $\left\langle x^{\prime} w^{\prime}\right\rangle$ within the metallic foil. Applying Eq. (A2), Eq. (A4), and Eq. (A8), we first calculate

$$
\begin{aligned}
\left\langle x w^{\prime}\right\rangle(z)= & \left\langle\sum_{i=1}^{n} \sum_{k=1}^{n} \Delta_{k}\left[A_{i} f_{i}\left(z_{k}-z_{i}\right)+B_{i} g_{i}\left(z_{k}-z_{i}\right)\right]\right. \\
& \left.\times\left[H\left(z_{k}-z_{i}\right)-H\left(z_{k}-z_{i+1}\right)\right] \delta\left(z-z_{k}\right)\right\rangle \\
= & \left\langle\frac{1}{2} \sum_{i=1}^{n} \Delta_{i} \delta\left(z-z_{i}\right)\left(\mathcal{A}_{i}+\sum_{j=1}^{i-1} \mathcal{C}_{i, j} \Delta_{j}\right)\right\rangle
\end{aligned}
$$

Because the $\Delta_{i}$ are isotropically distributed, we have $\left\langle\sum_{i=1}^{n} \Delta_{i} \delta\left(z-z_{i}\right) \mathcal{A}_{i}\right\rangle=0,\left\langle\sum_{i=1}^{n} \Delta_{i} \delta\left(z-z_{i}\right) \sum_{j=1}^{i-1} \mathcal{C}_{i, j} \Delta_{j}\right\rangle=$ 0 . In this result, note that for $j<i, \Delta_{i} \neq \Delta_{j}$ and all terms in the average vanish because there is no quadratic terms in $\Delta_{i}^{2}$ in the sums. Together, these results show that

$$
\left\langle x w^{\prime}\right\rangle(z)=0
$$

Similarly, we compute $\left\langle x^{\prime} w^{\prime}\right\rangle$ within a metallic foil,

$$
\begin{aligned}
\left\langle x^{\prime} w^{\prime}\right\rangle(z)= & \left\langle\sum_{i=1}^{n} \sum_{k=1}^{n} \Delta_{k}\left[A_{i} f_{i}^{\prime}\left(z_{k}-z_{i}\right)+B_{i} g_{i}{ }^{\prime}\left(z_{k}-z_{i}\right)\right]\right. \\
& \left.\times\left[H\left(z_{k}-z_{i}\right)-H\left(z_{k}-z_{i+1}\right)\right] \delta\left(z-z_{k}\right)\right\rangle \\
= & \left\langle\frac{1}{2} \sum_{i=1}^{n} \Delta_{i}^{2} \delta\left(z-z_{i}\right)\right\rangle .
\end{aligned}
$$

$\left\langle\sum_{i=1}^{n} \Delta_{i}^{2} \delta\left(z-z_{i}\right)\right\rangle$ in Eq. (A11) is approximated by averaging it over a mean free path $\delta z$. Because the $\Delta_{i}$ are normal distributed centered on 0 with variance $G^{2} / \mathcal{E}_{b}^{2}(z) \delta z$, carrying out this average gives

$$
\begin{aligned}
\left\langle\sum_{i=1}^{n} \Delta_{i}^{2} \delta\left(z-z_{i}\right)\right\rangle & \simeq \frac{1}{\delta z} \int_{z-\frac{\delta z}{2}}^{z+\frac{\delta z}{2}} d z\left\langle\sum_{i=1}^{n} \Delta_{i}^{2} \delta\left(z-z_{i}\right)\right\rangle \\
& \simeq \frac{1}{\delta z}\left\langle\Delta_{n}^{2}\right\rangle \simeq \frac{G^{2}}{\mathcal{E}_{b}^{2}}
\end{aligned}
$$

resulting in

$$
\left\langle x^{\prime} w^{\prime}\right\rangle(z)=\frac{1}{2} \frac{G^{2}}{\mathcal{E}_{b}^{2}} .
$$




\section{APPENDIX B: LOADING IN THE RADIAL DIRECTION OF A BEAM WITH A RADIAL GAUSSIAN PROFILE ON THE $(r-z)$ GRID}

Transversely, the beam macroparticles are initially loaded as if the beam radial distribution is axisymmetrically uniform, following the uniform beam radial density

$$
\rho_{\mathrm{b}, \text { uni }}(R)= \begin{cases}\frac{\lambda}{\pi} \frac{1}{r_{b}^{2}}, & 0 \leq R \leq r_{b}, \\ 0, & r_{b}<R .\end{cases}
$$

The number of particles therefore scales as $R^{2}$ in the $(R-z)$ grid. Then, the radial coordinate $R$ of each of the macroparticles is mapped to the new coordinate $r$ to obtain a Gaussian distribution in the physical $(r-z)$ grid chopped at $r=r_{\max }$ using the formula

$$
\int_{0}^{r} \rho_{\mathrm{b}, \mathrm{gau}}(\tilde{r}) 2 \pi \tilde{r} d \tilde{r}=\int_{0}^{R} \rho_{\mathrm{b}, \text { uni }}(\tilde{R}) 2 \pi \tilde{R} d \tilde{R}
$$

where

$\rho_{\mathrm{b}, \mathrm{gau}}(r)= \begin{cases}\frac{\lambda}{\pi} \frac{1}{2 \sigma_{b}^{2}}\left[\exp \left(-\frac{r^{2}}{2 \sigma_{b}^{2}}\right) / \mathcal{N}\right], & 0 \leq r \leq r_{\max }, \\ 0, & r_{\max }<r\end{cases}$

is the Gaussian radial density chopped at $r_{\max } \cdot \mathcal{N}=1-$ $\exp \left[-r_{\max }^{2} /\left(2 \sigma_{b}^{2}\right)\right]$ is a normalization factor to account for the chopping. Equation (B2) yields an explicit expression for

$$
r=\sigma_{x} \sqrt{-2 \ln \left[1-\left(\frac{R}{2 r_{b}}\right)^{2} \mathcal{N}\right]} .
$$

Note that $r_{b}=2 \sigma_{b}$ as both values are rms-equivalent beam radii [6].

[1] M. Reiser, Theory and Design of Charged Particle Beams (John Wiley \& Sons, New York, 1994).

[2] J. J. Barnard and S. M. Lund, in Beam Physics with Intense Space-Charge, Hampton, VA, 2015, https://people.nscl .msu.edu/ lund/uspas/bpisc_2015/.

[3] C. L. Olson, Cone focusing of intense relativistic electron beams, Phys. Fluids 16, 529 (1973).

[4] J. Hasegawa, K. Kondo, Y. Oguri, and K. Horioka, Ion beam focusing with cone optics for WDM experiments, Nucl. Instrum. Methods Phys. Res., Sect. A 733, 32 (2014).

[5] R. F. Fernsler, R. F. Hubbard, and S. P. Slinker, Foil focusing of electron beams, J. Appl. Phys. 68, 5985 (1990).

[6] S. M. Lund, R. H. Cohen, and P. A. Ni, Envelope model for passive magnetic focusing of an intense proton or ion beam propagating through thin foils, Phys. Rev. ST Accel. Beams 16, 044202 (2013).

[7] E. Henestroza, B. G. Logan, and L. J. Perkins, Quasispherical fuel compression and fast ignition in a heavy-ion-driven x-target with one-sided illumination, Phys. Plasmas 18, 032702 (2011).

[8] G. Caporaso, S. Sampayan, Y.-J. Chen, J. Harris, S. Hawkins, C. Holmes, M. Krogh, S. Nelson, W. Nunnally, A. Paul et al., Compact accelerator concept for proton therapy, Nucl. Instrum. Methods Phys. Res., Sect. B 261, 777 (2007).

[9] J. Barnard, J. Armijo, D. Bailey, A. Friedman, F. Bieniosek, E. Henestroza, I. Kaganovich, P. Leung, B. Logan, M. Marinak et al., Ion beam heated target simulations for warm dense matter physics and inertial fusion energy, Nucl. Instrum. Methods Phys. Res., Sect. A 606, 134 (2009).

[10] A. Macchi, M. Borghesi, and M. Passoni, Ion acceleration by superintense laser-plasma interaction, Rev. Mod. Phys. 85, 751 (2013).

[11] S. Wilks, A. Langdon, T. Cowan, M. Roth, M. Singh, S. Hatchett, M. Key, D. Pennington, A. MacKinnon, and R. Snavely, Energetic proton generation in ultra-intense lasersolid interactions, Phys. Plasmas 8, 542 (2001).

[12] P. Ni, B. Logan, S. Lund, N. Alexander, F. Bieniosek, R. Cohen, M. Roth, and G. Schaumann, Feasibility study of the magnetic beam self-focusing phenomenon in a stack of conducting foils: Application to tnsa proton beams, Laser Part. Beams 31, 81 (2013).

[13] P. A. Ni, S. M. Lund, C. McGuffey, N. Alexander, B. Aurand, J. J. Barnard, F. N. Beg, C. Bellei, F. M. Bieniosek, C. Brabetz et al., Initial experimental evidence of self-collimation of target-normal-sheath-accelerated proton beam in a stack of conducting foils, Phys. Plasmas 20, 083111 (2013).

[14] G. Penn and J. Wurtele, Beam Envelope Equations for Cooling of Muons in Solenoid Fields, Phys. Rev. Lett. 85, 764 (2000).

[15] K.-J. Kim and C.-X. Wang, Formulas for Transverse Ionization Cooling in Solenoidal Focusing Channels, Phys. Rev. Lett. 85, 760 (2000).

[16] J. F. Ziegler, M. Ziegler, and J. Biersack, SRIM the stopping and range of ions in matter, Nucl. Instrum. Methods Phys. Res., Sect. B 268, 1818 (2010).

[17] A. Friedman, R. H. Cohen, D. P. Grote, S. M. Lund, W. M. Sharp, J.-L. Vay, I. Haber, and R. A. Kishek, Computational methods in the warp code framework for kinetic simulations of particle beams and plasmas, IEEE Trans. Plasma Sci. 42, 1321 (2014).

[18] J. D. Jackson, Classical Electrodynamics, 3rd ed. (John Wiley \& Sons, New York, 1998).

[19] M. J. Berger, J. Coursey, M. Zucker, and J. Chang, Stopping-Power and Range Tables for Electrons, Protons, and Helium Ions (NIST Physics Laboratory, Gaithersburg, MD, 1998).

[20] R. Hubbard, S. Goldstein, and D. Tidman, in Heavy Ion Fusion Workshop (Lawrence Berkeley National Laboratory, Berkeley, CA, 1979), p. 488.

[21] M. J. Berger, J. S. Coursey, M. A. Zucker, and J. Chang, ESTAR, PSTAR, and ASTAR: Computer programs for calculating stopping-power and range tables for electrons, protons, and helium ions, NIST, Physical Measurement Laboratory (1992).

[22] S. M. Lund and B. Bukh, Stability properties of the transverse envelope equations describing intense ion beam transport, Phys. Rev. ST Accel. Beams 7, 024801 (2004). 